\title{
Incidental discovery of an ascending aortic thrombus: Should this patient undergo surgical intervention?
}

\author{
Subhasis Chatterjee, MD, ${ }^{a}$ Susan S. Eagle, MD ${ }^{b}$ David H. Adler, MD, ${ }^{c}$ and John G. Byrne, MD, ${ }^{a}$ Nashville, \\ Tenn
}

Aortic mural thrombus has been recognized in $9 \%$ of patients presenting with arterial thromboembolism. ${ }^{1}$ With the use of computed tomographic (CT) analysis, magnetic resonance imaging (MRI), and transesophageal echocardiography (TEE), its recognition is increasing. Although most reported cases are diagnosed after an embolic event, incidental aortic thrombi will increasingly confront cardiovas-

From the Department of Cardiac Surgery, ${ }^{\mathrm{a}}$ the Department of Anesthesiology, ${ }^{\mathrm{b}}$ and the Division of Cardiovascular Medicine, ${ }^{\mathrm{c}}$ Vanderbilt Heart \& Vascular Institute, Nashville, Tenn.

Disclosures: None.

Received for publication June 9, 2009; accepted for publication July 5, 2009; available ahead of print Aug 19, 2009.

Address for reprints: John G. Byrne, MD, Vanderbilt Heart and Vascular Institute, 1215 21st Ave South, Medical Center East 5th Floor South Tower, Nashville, TN 37232-8802 (E-mail: john.byrne@vanderbilt.edu).

J Thorac Cardiovasc Surg 2010;140:e14-6

$0022-5223 / \$ 36.00$

Copyright (c) 2010 by The American Association for Thoracic Surgery

doi:10.1016/j.jtcvs.2009.07.010 cular specialists. We present a rare case of an incidental aortic mural thrombus and discuss its management.

\section{CLINICAL SUMMARY}

A 36-year-old man with a history of hypertension, smoking, and cocaine use presented with chest pain radiating into his left arm. An electrocardiogram demonstrated sinus rhythm with early repolarization. Cardiac enzyme levels were increased, with a troponin I value of $1.20 \mathrm{ng} / \mathrm{mL}$ (nor$\mathrm{mal},<0.05 \mathrm{ng} / \mathrm{mL}$ ). Results of urine toxicology were positive for cocaine. He was treated with aspirin, clopidogrel, and a heparin infusion for a non-ST-segment elevation myocardial infarction. A CT angiogram to exclude aortic dissection demonstrated a filling defect in the ascending aorta (Figure 1, A). An MRI/magnetic resonance angiogram showed a small subendocardial inferoapical myocardial infarction and a polypoid lesion in the midascending aorta connected by a thin stalk (Figure 1, B). TEE showed an 8


FIGURE 1. Diagnostic imaging: A, computed tomographic angiogram of the ascending aortic mass; B, magnetic resonance image/magnetic resonance angiogram demonstrating a filling defect; $\mathrm{C}$, transesophageal echocardiogram showing a mobile mass; $\mathrm{D}$, 3-dimensional transesophageal echocardiographic image. 


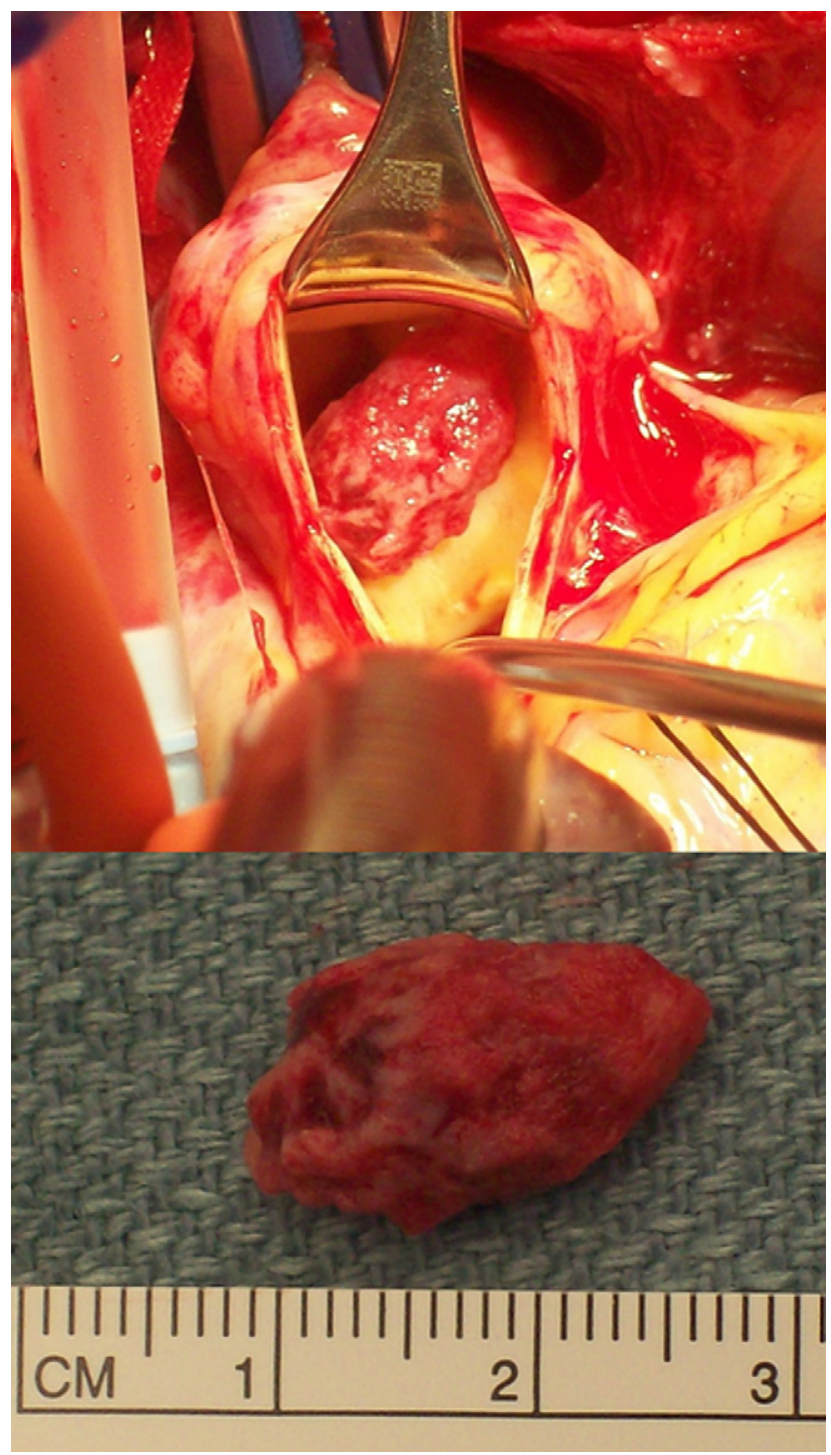

FIGURE 2. Intraoperative photographs: A, intraoperative view of the aortic mass; B, removed aortic thrombus.

$\times 19-\mathrm{mm}$ mobile ascending aortic mass and no other wall motion or valvular abnormalities (Figure 1, C).

He was asymptomatic, and his coronary event was believed to be related to cocaine use. At this point, it was discussed whether the mass should be treated with anticoagulation alone, elective surgical resection, or emergency surgical resection. The team of cardiologists and cardiac surgeons determined that surgical intervention would be best.

The patient was taken for surgical intervention the following morning. The femoral artery was cannulated, and a dual-stage venous cannula was placed in the right atrium. Intraoperative 3-dimensional TEE demonstrated the mobile mass (Figure 1,D), and epiaortic ultrasonographic scanning demonstrated a safe region to clamp the aorta to avoid circulatory arrest. After initiation of cardiopulmonary bypass, the proximal ascending aorta was opened transversely. The mass was found to be attached by a thin stalk to the anterior surface of the aorta at the level of the right pulmonary artery (Figure 2). This was removed without difficulty. There was no evidence of endocarditis noted on the tricuspid aortic valve, and the ascending aorta was normal without intimal defect or atheroma. The patient was easily weaned from cardiopulmonary bypass and was transported to the intensive care unit. His hospital course was unremarkable, and he was discharged to home on day 5 with aspirin therapy because his compliance with warfarin was believed to be unreliable. Pathology showed a pink-red $2.5 \times 1.1-\mathrm{cm}$ benign organizing thrombus (Figure 2).

\section{DISCUSSION}

Primary free-floating aortic mural thrombus is a less common cause of emboli compared with emboli from superimposed thrombus on an atherosclerotic plaque. Many risk factors, including atherosclerosis, dissection, malignancy, and hypercoagulable states, have been associated with aortic mural thrombi.

CT is the most widespread screening imaging modality and provides valuable information regarding diagnosis, precise localization, and extent of the thrombus. MRI can help differentiate thrombus from tumor. The advantages of TEE are that it can be promptly performed without any exposure to contrast agents with a high degree of accuracy. TEE limitations include the inability to visualize portions of the aortic arch. Either of these modalities or their combination is suitable for diagnosis.

There is no consensus on the management of a mobile aortic thrombus discovered after embolization. Treatment modalities have included heparin/warfarin alone, ${ }^{2}$ thrombolysis, ${ }^{3}$ and surgical intervention. In the largest reported series Choukroun and colleagues ${ }^{4}$ reviewed the management of 9 embolic cases (4 patients presented with upper extremity embolization and 5 with stroke), which were diagnosed by means of TEE. The authors recommend routine heparin anticoagulation for 2 weeks followed by repeat TEE. At that time, if the thrombus disappears, then warfarin therapy is started. If the thrombus is smaller, heparin is continued. Surgical intervention is considered if heparin therapy is ineffective. However, each of the cases involved either the aortic arch or descending aorta, with an overall success rate of $44 \%$ with anticoagulation alone.

Nevertheless, anticoagulation itself poses risks, including bleeding into atherosclerotic plaques and plaque rupture. Anticoagulation might also prevent the protective fibrin layer over an ulcerated aortic plaque, which stabilizes the plaque, preventing embolization. Nonetheless, a free-floating aortic thrombus in the ascending aorta can be considered an important embolic risk. ${ }^{5}$ Our recommendation is prompt surgical intervention to prevent catastrophic embolization. Although a left ventricular thrombus can be treated with 
anticoagulation alone in some cases, we believe that the embolic potential of a mobile ascending aortic thrombus warrants prompt intervention.

\footnotetext{
References

1. Reber PU, Patel AG, Stauffer E, Muller WF, Do DD, Kniemeyer HW. Mural aortic thrombi: an important cause of peripheral embolization. JVasc Surg. 1999;30:1084-9.

2. Blackshear JL, Jahangir A, Oldenburg WA, Safford RE. Digital embolization from plaque-related thrombus in the thoracic aorta: identification with transesophageal
}

echocardiography and resolution with warfarin therapy. Mayo Clin Proc. 1993; 68:268-72.

3. Hausmann D, Gulba D, Bargheer K, Niedermeyer J, Comess KA, Daniel WG. Successful thrombolysis of an aortic-arch thrombus in a patient after mesenteric embolism. N Engl J Med. 1992;327:500-1.

4. Choukroun EM, Labrousse LM, Madonnal FP, Deville C. Mobile thrombus of the thoracic aorta: diagnosis and treatment in 9 cases. Ann Vasc Surg. 2002;16: 714-22.

5. Madershahian N, Kuhn-Regnier F, Ben Mime L, Slottosch I, Langebartels G, Sindhu D, et al. A loose cannon: free-floating thrombus in ascending aorta. $J$ Card Surg. 2008;24:198-9.

\title{
Mechanical left ventricular unloading to prevent recurrent myocardial rupture
}

\author{
Stephen Westaby, MS, PhD, ${ }^{\mathrm{a}}$ Vipin Mehta, MD, ${ }^{\mathrm{a}}$ Fidelma Flynn, MD, ${ }^{\mathrm{b}}$ and Neil Wilson, $\mathrm{MD},{ }^{\mathrm{c}}$ Oxford, \\ United Kingdom
}

Temporary left ventricular assist devices (LVADs) are used to sustain systemic blood flow during acute left ventricular (LV) failure. Ventricular unloading also reduces intracavity pressure and wall tension. Decreased LV work might then allow functional recovery after postischemic stunning or viral myocarditis. ${ }^{1}$ Extrapolating from this, LV unloading could be used to protect against myocardial rupture in injured hearts with normal contractility. This strategy is illustrated by the following case.

\section{CLINICAL SUMMARY}

An otherwise healthy 15-year-old (44-kg) girl presented with a 3-week febrile illness and large pericardial effusion. Blood cultures grew Staphylococcus aureus. During hospital investigation, she experienced sudden cardiovascular collapse and pulmonary edema. Echocardiographic analysis showed severe mitral regurgitation, with large mobile vegetations filling the small left atrium. Requiring intermittent cardiac massage, she was bought to the operating room (OR). Through a median sternotomy, the $800-\mathrm{mL}$ bloodstained effusion was drained, and cardiopulmonary bypass was established. The left atrium was approached by means

\footnotetext{
From the Department of Cardiac Surgery, ${ }^{a}$ Nuffield Department of Anaesthetics, ${ }^{b}$ and Department of Paediatric Cardiology, Oxford Children's Hospital, ${ }^{\mathrm{c}}$ John Radcliffe Hospital, Oxford, Untied Kingdom.

This mechanical circulatory support programme is supported by the Gyde Trust.

Disclosures: None.

Received for publication June 17, 2009; accepted for publication July 5, 2009; available ahead of print Aug 31, 2009

Address for reprints: Stephen Westaby, MS, PhD, Department of Cardiothoracic Surgery, John Radcliffe Hospital, Headley Way, Headington, Oxford OX3 9DU, United Kingdom (E-mail: swestaby@ahf.org.uk)

J Thorac Cardiovasc Surg 2010;140:e16-7

$0022-5223 / \$ 36.00$

Copyright (c) 2010 by The American Association for Thoracic Surgery doi:10.1016/j.jtcvs.2009.07.009
}

of superoseptal incision. A 4-cm $\times 2$-cm pedunculated vegetation was removed, revealing dehiscence of the posterior mitral commissure with flail anterior and posterior leaflets. Beneath and separate from the annulus, there was ulceration of the endocardium with vegetations. The mitral valve was repaired by excising infected tissue and sewing an elliptical bovine pericardial patch into the annulus. The flail leaflets were then reattached to the patch. Vegetations were curetted from the infected LV myocardium. Intraoperative transesophageal echocardiographic analysis showed the reconstructed valve to be fully competent. Apart from temporary left-sided weakness, she made a straightforward recovery but had repeated febrile episodes.

On the seventh postoperative day, she again experienced sudden cardiovascular collapse with cardiac tamponade. On this occasion, echocardiographic analysis showed a tense posterior intrapericardial collection and an aneurysmal area at the site of the myocardial abscess. The repaired valve and mitral annulus remained intact (Figure 1). She was rapidly conveyed to the OR and started on cardiopulmonary bypass. Lifting the heart, a ruptured mycotic aneurysm of the left ventricle was identified between the circumflex marginal and distal circumflex branches. The disintegrating epicardium was debrided and closed with deep Teflon plegeted mattress sutures, avoiding the coronary branches. The following day during extubation, the ventricle ruptured again. Back in the OR, renewed attempts to close the leak occluded the dominant circumflex coronary, causing acute LV failure. Removal of the sutures restored contractility, but subsequent repair remained tenuous. To prevent further dehiscence, we used a centrifugal blood pump (Rotaflow, Maquet, Germany) to empty the left heart and reduce systolic LV wall tension. By using left atrial and ascending aortic cannulation, the LVAD was left in situ for 16 days pending control 